\title{
ÜBER DIE FORMEN DES KONJUNKTIVS IM BRITANNISCHEN.
}

Obwohl H. Ebel, als er, vor mehr als einem Menschenalter, das Verhältnis des Konjunktivs in den britannischen Dialekten untersuchte, die zuverlässigern Texte, deren wir uns heute erfreuen, entbehren musste, so konnte es dem ausgezeichneten Philologen doch nicht fehlen, dass er Irrtümer der Vorgänger schon damals aufdeckte und den Modus in gewisse unbestreitbare Rechte einsetzte. ${ }^{1}$ ) Einige Merkmale der Form entgingen ihm freilich, traten auch dann in seiner Ausgabe der Grammatica celtica nicht scharf genug hervor. Noch Atkinsons wertvolle Arbeit über den Modus Conjunctivus im Mittelwelschen hat es, wie ich in dieser Zeitschrift 3,153 andeutete, darin versehen, dass sie der ältern Sprache den Conjunctivus Imperfecti abspricht. ${ }^{2}$ ) Eine kürzlich erschienene Schrift von J. Vendryes ${ }^{3}$ ) stellt denn auch, wie sich gebührt, neben den Konjunktiv des Präsens carho 'amet' den des Imperfekts carhei 'anıaret'. Sie erkennt den letztern auch in den armorischen Dialekten, die

1) De verbi britannici futuro et coniunctivo. (Jahres-Bericht über das Städtische Progymnasium in Schneidemühl 1866, p. 3-8). Sein Ergebnis fasst der Verfasser p. 8 in die Worte: 'Concordant igitur omnes dialecti britannicae hac re, quod vera futuri specie carent; discordat aremorica a ceteris, quae praesentis forma futurum significant, coniunctivi propriam vim servarunt'.

2) Noch Griffith Roberts, a Welsh Grammar 1567, p. 157, kennt einen Conjunctivus Imperfecti, giebt ihm aber eine aus Präsens und Imperfekt gemischte Flexion: pann garun, garyt, garai; garom, garoch, garont.

8) De l'imparfait du subjonctif en moyen-gallois. (Mémoires de la Société de linguistique de Paris, Tome XI. 1900.) $10 \mathrm{pp}$. 
carfe oder careh̀̀ 'amaret' neben dem Indikative carè 'amabat' haben. Beifällig und mit ungeminderter Aufmerksamkeit begleitet man die Darlegung des Verfassers bis auf die letzte Seite, wo er den Ursprung dieser Formen und ihres $h$ bespricht. Das $h$ des mittelwelschen Konjunktivs ist nach seiner Vermutung das $s$ der irischen Futurbildung fortias von fortiagaim 'ich helfe'. Auch Thurneysen scheint eine solche Annahme zu begünstigen (KZ. 31,71. Brugmann 2,1299). Aber indem ich die Formen aufs neue zusammenhielt und überdachte, ist mir ein Zweifel gekommen. Jenes Futurum auf $s$ ist im Irischen, wie nun Strachans Prüfung aufs neue bestätigt, auf bestimmte Verbalauslaute beschränkt und schon im Absterben; das Welsche scheint nur ganz vereinzelte Reste davon bewahrt zu haben. So ist w. gwares 'er helfe' (von gwared) aus dem irischen fu-m-ré-se 'mihi succurrat' (von rethim) gedeutet worden. Auch w. duch 'ducat' BBC. $20 \mathrm{~b} 5$ (= arm. douc) hat man ähnlich erklärt (RC. 20,79). ${ }^{1}$ ) Vielleicht giebt es noch andere Beispiele; nämlich wie dem ir. doruacht, roacht, toracht 'pervenit' von der Wurzel ag- das welsche aeth 'ivit', daeth 'venit' an die Seite tritt, so dem ir. dorua, roa, ró, tora 'perveniet' das w. a, aa: arm. ay 'ibit', w. daw 'veniet' etc. Dass auch w. el 'ibit' ein solches Futur ist, kann ich nicht bestimmt behaupten (vgl. Stokes, Sprachschatz p. 43); es spricht dafür möglicherweise, dass el und seine Komposita in der ältern Sprache, wie Atkinson bemerkte, auch ohne die Endung -ho modal gebraucht wird. ${ }^{2}$ )

Von den Grundzügen altertümlicher Sprachbildung, deren das Irische einzelne bis in die Neuzeit behalten hat, ist dem Welschen aus vorbritannischer Zeit nur wenig übrig geblieben.

1) Es ist wohl möglich, dass duch eine sigmatische Form ist, einem ir. *dus entsprechend, für ${ }^{*}$ ducst, ${ }^{*} d u c s e t$; sowie ir. oss : w. ych ox; es- : achex; uas : uch ux-ellos; ses-, sé : chwech sex; dess : dehou $\delta \varepsilon \xi\llcorner\iota s ;$ tressa : trech 'fortior'; lassar : llachar 'igneus' etc. Die Form gehört zu dem w. Infinitive dygu (statt dwyn, arm. dougen) von dem celtischen Verbalstamm uc-, der auch in aduc 'abstulit', amuc 'defendit', goruc 'fecit' enthalten ist. Es ist aber zu bemerken, dass die dem duch 'ducat' analog gebrauchte Form von amygu Skene 149, 3 amwc 'protegat' lautet, was man als das Futurum zu dem Präteritum amwyth (S. Evans, Dict. 1, 204b) angesehen hat. Ist aber duch wirklich ein Konjunktiv auf -cs, so beweist diese Form allein schon, dass der gewöhnliche Konjunktiv dycco andern Ursprungs ist.

2) el 'eat' RB. 1, 14; del 'reniat' 1,34. 37. 221. 224. Skene 231, 1. 234, 10; gwnel 'faciat' RB. 1, 44. BBC. 20 b 4 . gunel 35 b 14. 
Indem die alten Verhältnisse sich lösten und die Analogieen sich lebhaft durcheinander drängten, entstanden hier grammatische Formen, deren wesentlicher Charakter Zusammensetzung ist. Man schuf Tempus- und Moduszeichen, in denen die Urbilder kaum noch zu erkennen sind. Man schuf Personalendungen, indem man selbst Pronomina und Partikeln nutzbar machte, wie carwn : arm. caromp 'amamus' aus caram-ni, carom-p-ni (wie contempno), cerwch 'amate' aus ceri-chwi, carut 'amabas' aus caru-ti, und sogar vielleicht, dem j'avons im Patois vergleichbar, ${ }^{1)}$ ein cerych 'ames' aus cery-chwi (schwerlich aus cery-ooı oder cery-sva) nicht scheute. Ein irisches Futur wie ni im-irchói 'er wird mich nicht schädigen' Wb. 7 a 11 (von erchoit 'Schaden') ist eine starke Formation von indogermanischer Ursprünglichkeit, aber ein welsches ergyttyo 'accedat' RB. 1, 103. 267 (von ergydyaw 'treffen' HM. 1, 235) steht auf der Stufe eines ir. erchotigea oder auf einer noch spätern. Sollte jene vergessene Bildung des sigmatischen Konjunktivs in der neuen Phase der Sprache wieder lebendig werden und ganz überraschend zunehmen, wo man von der altceltischen Flexion so vieles verloren und einige der nötigsten Endungen sogar dem Lateinischen entlehnt hat? Ich halte meine Zustimmung noch zurück, hoffend, es werde die umständliche Erörterung, der ich mich unterfange, das eine oder das andere ergeben, das ins Gewicht falle.

Aus der Formation des irischen Konjunktivs ist von vornherein nicht viel Aufklärung zu erwarten, da er (von den Resten beth 'esset', dogneth 'faceret', KZ. 27, 183. 31, 66, abgesehen) nur Praesentis ist. Die welschen Konjunktive erscheinen zunächst als ganz unabhängige Bildungen, deren gleichmässiger Charakter ein $h$ ist, im Präsens sowohl wie im Imperfektum und im Aktiv wie im Passiv; also in der 3. Sg. von caru 'lieben', tebygu 'meinen', gwybod 'wissen':

Praes. act. carho, tebycko (statt tebyg-ho), gwypo (statt gwyb-ho), zu dem Indikative car, tebyg, gwyr.

Imperf. act. carhei, tebyckei, gwypei, zu dem Indikative carei, tebygei, gwybydei, auch gwyddai (D. G. 102, 6 als Konjunktiv gebraucht).

1) Dergleichen kommt auch in andern Sprachen vor; z. B. sagt man im marokkanischen Dialekte des Arabischen nektub 'scribam' (eig. 'scribemus') statt ektub und unterscheidet nektub 'scribemus' davon durch eine plurale Endung: nektubî. 
Praes. pass. carher, tebycker, gwyper, zu dem Indikative cerir, tebygir, gwybydir (D. G. 188, 26. 50) oder gwyddir.

Imperf. pass. cerhit, tebyckit, gwypit, zu dem Indikative carwyt, tebygwyt, gwybuwyt; ebenso adnepit RB. 1,70 von. adnabod 'erkennen'.

Die Belege sind so zahlreich, dass die Richtigkeit dieser Aufstellungen nicht in Frage kommt, wenn man das $h$ der Konjunktive selbst in den alten Texten, die wir haben, hin und wieder vermisst, wie es denn im Neuwelschen fast gänzlich ausser Gebrauch getreten ist. ${ }^{\text {) }}$ Auch ist die Bedeutung dieses Buchstaben, die Natur seines Lautes derart, dass er sich oftmals eindrängt, wo er nicht hingehört, z. B. ny chanhwyt 'non cantum est' Skene 145, 15. Das lasse ich unbeachtet.

Das $h$ der Konjunktive findet sich nach jedem Stammauslaute. Nach Vokalen: aho 'eat' RB. 1, 140, gwnaho 'faciat'

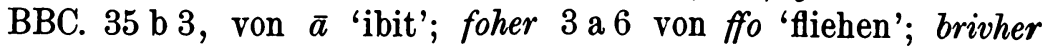
3 a 5 von briwo 'brechen'; clyho Skene 114, 2, clywhont RB. 1,14, clywher Skene 296, 14 (neben clywir ib. 20), von clybod 'hören'; dylyho RB. 1,257. LA. 45, 13 von dylyu 'verdienen'; dalhyo RB. 1, 123, dalyher LA. 45, 27, von daly 'fassen'; cudyho LA. 40, 17 von cuddio 'verbergen'; coffaho BBC. 35 b 8 von coffau 'erinnern'; bwyttehych RB. 1, 292 von bwyta 'essen'. Dass die Denominative auf -hau das konjunktivische $h$ sonst vermeiden, habe ich schon bemerkt, also parhawyf RB. 1,24, parhao 1,213, bwyttao 1,289, rwydhao 1,17 , rithao $\mathrm{BBC}$. 46 b 11, rydhaer $\mathrm{RB} .1,65$.

1) Griffith Roberts war der Konjunktivcharakter wohl bekaunt, denn er schreibt in seiner Grammatik p. 157 vor, dass man bilde: pann grettuyf von credu, pann dybyccuyf von tybygu und pann gyphlyppuyf von cyphlybu. Wie das Verständnis der Form allmählich verloren ging, dafür liefert Gruffydd von Hiraethog, ein Barde in der ersten Hälfte des 16. Jahrh., ein Beispiel. Er schliesst sein Gedicht 'über die Eifersüchtige' mit den Versen:

Y mwya' ei dig a'i ddigedd,

Ei bwrn a'i dycco i'r bedd.

'Die sich und ihn am meisten ärgert, möge ihre Bürde sie ins Grab bringen!' Und in demselben Gedichte sagt er, der Allitteration zu liebe:

Ffei o wraig wych ffriw a gwedd

A ddygo fwrn o eiddigedd.

'Pfui über eine Frau, hübsch von Ansehn und Manier, die eine Bürde der Eifersucht trägt!' 
Nach den Liquiden $L, R, N$, wie in: talho BBC. 4 a 11 , talhont Skene 128, 4, von talu 'bezahlen'; celho BBC. 4 a 4 . Skene 213 von celu 'verhehlen'; guelher BBC. 1 b 6 von gweled 'sehen'; ae dehoglho 'qui id interpretetur' 4 a 4; elhont RB. 1, 61, elhei 1, 85, elhynt 1, 111, von el 'ibit'; delhich BBC. 42 b 7, delhei RB. 1, 109, delher 1,61, von del 'veniet'; gwnelhoch 1,140, gwnelhont BBC. 30 b 4, gwnelhit RB. 1, 105, von gwnel 'faciet'; - carho RB. 1,252 (neben kerych 1, 18. 264) von caru 'lieben'; sorho Skene 157,9 (neben sorro BBC. $28 \mathrm{~b} \mathrm{1}$ ) von sorri 'zürnen'; - canho BBC. 42 b 5. Skene 129, canhont BBC. 17 a 6 , cenhid 5 b 2, von canu 'canere'; mynhwyf Skene 193, 16, mynho 206,2, mynhont Cymmr. 9,62, mynhei RB. 1, 277, mynhut 1, 213, von mynnu 'wünschen'; tynho 1,222 (neben tyno 1,110) von tynnu 'ziehen'; prynhom Skene 116,26, prinhei BBC. 21 a 1, von prynu 'kaufen'; erlinho 4 b 8 von erlyn 'verfolgen'; digonho RB. 1, 120, digonhom BBC. 15 b 3, von digoni 'genügen'; llunhich $42 \mathrm{~b} 7$ von llunio 'bilden'; gwaravunho RB. 1, 253 (ohne $h$ 1,263) von gwarafun 'verhindern'; barnher Skene 158, 22. RB. 1, 256 von barnu 'richten'. Nach den Spiranten $D D$ und $F$, wie in: lladho RB. 1, 15. 189, ymladho Skene 239,20, llather (für llad-her) BBC. 29 a 12, von lladd 'töten'; dygwydho Skene 231, 15 von dygwyddo 'fallen'; eistetho (für eistedho) BBC. $29 \mathrm{~b} 4$ von eistedd 'sitzen'; rodho LA. $113,30=$ rotho $102,20.130,1$ (neben rodo $\mathrm{RB} .1,222=$ roto BBC. 15 a 12), rodhom $\mathrm{RB} .1,105$, rothei (für rodhei) BBC. 22 a 3, rodher RB. 1,258, von roddi 'geben'; nodho 1,126 von noddu ,bitten'; - yfho RC. 8,21 von yfed 'trinken'; safhei RB. 1,110 von sefyll 'stehen'; prouher BBC. 3 b 1 von profi 'probare'.

Nach den Mediae $G, D, B$, wie in: tebycko RB. 1, 125, tebyckych 1,120, tebyckwn 1,165, tebycher Skene 225, von tebygu 'meinen'; bendicco 161, 24 (neben bendigwyf 292,10) von bendigo 'segnen'; plyccoent Cymmr. 9,62 von plygu 'biegen'; mynaccont LA. 168, 7 von menegi 'erzählen'; diwyccom BBC. 15 b 3, dywyccviff 42 a 15, von diwygu 'wiederherstellen'; dirmycco 35 b 9 von dirmygu verachten'; gostecker RB. 1, 280 von gostegu 'gebieten'; llosco Skene 120,10 von llosgi 'brennen'; kysco RB. 1,122 von cysgu 'schlafen'; gwascut 1, 116 von gwasgu 'drücken'; dyckwyf LA. 79, 28, dycko RB. 1, 109. 124. 127, dycco D. G. 149, 34, dazu an duch 'er bringe uns' BBC. 20 b 5, von dug- (dwyn) 'bringen'; cretto RB. 1, 189, crettoch 1,131, von credu 'glauben'; notto 1, 105. 118. 120.126, nottych 1, 106, von nodi 'bezeichnen'; dywetto 
1,217. 222, dywettych 1, 113. 276, dywettei 1,237, dywetter Skene 242, 2. D. G. 240,41 , dywettit RB. 1, 94, von dywedyd 'sagen'; diaspettych 1,114 von diaspedain 'schreien'; retto Skene 240,11, rettei RB. 1, 108. 262, von rhedeg 'laufen'; gattwyf Skene 168,28, gatto D. G. 114, 56, gatter RB. 1, 113.119, von gadu 'verlassen'; dotter RB. 1, 15. 57. 123. D. G. 114, 51 (neben dodir 119, 50), dottit 1, 13. 153, von dodi 'geben'; gwatter BBC. 51 a 14 von gwadu 'leugnen'; ergyttyo RB. 1, 103, erkyttyo 1, 267 von ergydio 'erreichen'; cattwo 1, 123. 177. D. G. 130, 33 von cadw 'hüten'; atteppych RB. 1, 176 von attebu 'antworten'.

Solche Formen liegen vor und sie lassen sich leicht vermehren; ihr gemeinsamer Charakter verbirgt sich uns unter dem vieldeutigen $h$. Dass er ursprünglich überall ein $s$ gewesen sei, lässt sich, soviel ich sehe, nicht wahrscheinlich machen. Wenn wir die welschen Konjunktive jeder Art zu verstehen suchen, ihre Formen bis in die ältesten Denkmäler zurückverfolgen, uns bei den verwandten Dialekten Rats erholen, je mehr wir auf den Gegenstand eingehen, desto verwickelter und schwieriger gestaltet er sich.

Eine Frage muss, ehe wir irgend etwas entscheiden, zu allererst beantwortet werden. Kommt dieses $h$, oder auch seine Wirkung, einen vorhergehenden Wurzellaut zu verhärten, in der Konjugation des Verbum substantivum vor?

Der Austausch der Meinungen, der stattgefunden hat (KZ. 26, 423. 27, 165. 28, 55), scheint mir zu ergeben, dass die celtischen Sprachen zwei mit $b$ anlautende Warzeln für das Verb 'sein' verwenden, die in ihren Urformen ähnlich sind, aber doch ziemlich weit auseinander liegen, nämlich: sanskr. jîvâmi, ßıóo, vīvo, air. biuu, und sanskr. bhavâmi, $\varphi v i o$, ahd. bim, wozu lat. fuit und air. bói in gleicher Bedeutung gehören. Wie auf diese beiden Wurzeln die Formen der britannischen Dialekte zu verteilen sind, ist keineswegs leicht festzustellen. Schon darüber sich zu einigen ist schwer, ob das altwelsche bid, mittelwelsche byd, neuwelsche bydd, cornische byth, armorische bez zu der ersten Wurzel $B \hat{I}(B E I)$ oder zu der zweiten $B U$ gehört? Ist byd 'sei' = ir. bi 'vive' oder $=$ ir. $b a$ (statt bav) 'fi'? und ist byd 'er ist, wird sein' = ir. bi 'vivit', bia 'vivet' oder = ir. bid, ba 'fiet'? Es ist bekannt, dass byd, dessen Verständnis Schleicher vergeblich im Slawischen suchte (KB. 1,505. 5, 318), als ein j-Stamm erklärt worden ist, wie er im äolischen yvio zu Tage liegt (RC. 2,116). 
Ist das wahrscheinlich? Bedenklich erscheint von vornherein jede Erklärung einer celtischen Wortform, die nicht im Celtischen selbst ihren Grund und ihre Stütze hat; man dürfte diesem Sprachzweige getrost jenen Leitsatz zueignen, den einst Jacob Grimm für den seinigen aufgestellt hat. 'Bei unsern deutschen Wörtern', so sagt er in der Vorrede des Wörterbuchs, 'muss es recht sein vor allem zu versuchen, ob sie nicht auch innerhalb dem deutschen Gebiet selbst sich erklären lassen, das zwar nur engere, der Natur der Sache nach oft sichrere Schritte zu thun erlaubt.' Muss man nicht glauben, dass, wie sich w. $d y d$ (alt did) : arm. dez 'Tag' zu ir. die, dia 'dies' (aus dives) verhält, so sich auch verhalte w. byd (alt bid) : arm. bez 'sei, ist' zu ir. bi 'vive, vivit' oder bia 'vivet'? Wie neben $d y d$ eine Form $\left.d y w,{ }^{1}\right)$ pl. diev BBC. $28 \mathrm{~b} 11$ : arm. de, pl. deiou, liegt, so neben byd das Adjektiv w. byw : corn. bew : arm. beu = ir. biu, béo 'vivus' etc. Wird nicht ebenso das ir. sniim 'flechte' im Welschen zu nyddaf? Analog sind ferner trydydd tritîya 'dritt', rhydd 'frei', blydd 'zart', toddi 'tauen'. W. ydd geht meist auf ir. idh zurück (vgl. ffydd, cybydd, crefydd, gwydd, hydd), und eigentlich ist auch bydd nicht anders zu verstehen als ein neuir. bidh für bi oder vielmehr bij, biv. So ist auch w. Ywerydd 'Irland' aus dem ir. Ériu zu erklären (Skene 2,355. Sprachschatz p. 45), wovon der casus obliquus Ywerddon = ir. Érend, Érind (wie w. afon = ir. abha, abhand). Neben henwyf 'existo' giebt es auch ein handwyf Skene 265, 17, und es scheint, als habe das $i$ der ursprünglichen Form *sani (ir. sain) in der erstern Form Umlaut bewirkt, in der andern aber sich zu $d d$ verdichtet. Die 3. sg. handit 'existit' Skene 115, 16. 270,7. RB. 1, 178 oder handid 1, 71 (gleichsam ir. sain-atá) ist bekannt. Ähnlich wird es sich mit hudwyf Skene 144, 26 (MA. 33 a) verhalten, neben hubwyf. Für die Präpositionen $i, y$ 'zu' (ir. do) und $o$ 'von' (ir. ó) hat der südwelsche Dialekt vor Vokalen die Formen idd und odd, und w. iddo : corn. dozo : arm. dezaf 'ihm' (ir. dó) etc. muss vielleicht aus diesem idd erklärt werden, obwohl erddo, hebddo, rhagddo etc. eher an den

1) Die Form $d y w$ ist in den Namen der Wochentage gebräuchlich, daher in alten Texten wie das Wort für 'Gott' geschrieben: duw Skene 158. 173. 189. 207. 222. 270. 298 oder diw 83. 105. Statt duwieu 'Donnerstag' Skene 207, wo die Aussprache einen konsonantischen Ruhepunkt suchte, ist gewöhnlicher diuieu Skene 301, 3 oder dyv-iev BBC. 23 b 4 oder dyfieu Skene 158. $170-$ ein Zeichen der Verwandtschaft zwischeu $d d$ und $v$, 
Ursprung dieses $d d$ aus dem Artikel ind denken lassen. Halb ein Konsonant und halb ein Vokal, erscheint und verschwindet dies w. $d d$ mitunter auf unberechenbare Weise; man denke an w. oed 'er war' $=$ arm. oa, w. oedwn 'ich war' $=$ arm. oan; w. gwdost 'du weisst' neben gwybuost 'du wusstest' von gwybot st. gwydbot, wie nw. gwybed 'Fliegen' = mw. gwydbet RB. 1, 54 . 112. LA. 10, 23, nw. gwybwyll = mw. gwydbwyll RB. 1,84. 220 . $235=$ ir. fidchell; nw. rhoddi 'geben' = rhoi, arm. rei (KZ. 30, 221); arm. mezeven 'Mittsommer' $=$ vann. meheüenn $=\mathrm{w}$. mehefin (RC. 16, 189) = ir. midh-shamhain, meitheamh; narm. délez 'Raa' = marm. delé, u. a. m. ${ }^{1}$ )

1) Für die Geschichte des welschen $d d$, worüber noch viel zu sagen wäre, ist auch das Wörtchen ydd von Wichtigkeit. Das w. issid, yssyd, sydd, sy scheint doch nicht, wie ich CZ. 3,149 auch angenommen habe, das ir. ished, iseadh zu sein, da man hierfür eher w. *issed erwarten sollte und auch die Bedeutung nicht ganz übereinstimmt. Ir. ished ist eine Kopula, die in der Regel an der Spitze des Satzes steht, franz. 'c'est' und ihre Stelle nimmt das w. isem GC. 398, yssef Skene 148, $32=$ sef ein: altw. issem $i$ anu 'das ist sein Name' (KB. 7, 400) = ir. ished a ainm. Dagegen ist yssyd eigentlich ein Wort des Daseins, das selten voransteht, franz. ' $y$ est', 'qui y est'; z. B. nifer $a$ uu ac a uyd, vch nef is nef meint yssyd 'viele waren und werden sein, über dem Himmel, unter dem Himmel giebts eine Menge', Skene 114, 15, ähnlich 292, $34 \mathrm{f}$.; dann auch als Relativpronomen: aw. ir hinn issid Crist 'dieser, welcher ist Christus', KB. 4, 411. Nach meinem Vermuten ist issid, yssyd das ir. is and 'es ist da'; denn dieses aw. $i d, \mathrm{mw} . y d, \mathrm{nw} . y d d$ $=$ ir. and dient auch zur Verstärkung des Pronomen demonstrativum, wie in henoid 'cette nuit-ci' (Iuv.); ir gur hunnuid im Martianus, d. i. y goor hwnnw$y d d$ 'dieser Mann da' (KB. 7, 390), of. arm. hennez 'iste'. Vielleicht ist dieses $i d, y d, y d d$ die Verbalpartikel $y d$, die vor Konsonanten das $d$ abwirft, vor Vokalen aber in der neuern Sprache (mit häufiger vorkommendem Lautwechsel) $y v$ lautet. Sie scheint eigentlich aus yssyd verkürzt zu sein, denn w. $y$ dywawt 'er sprach da' ist $=$ ir. is and asbert, wofür im Mittelirischen auch ised asbert gesagt wird $=$ w. sef $y$ dywawt. Selten steht ir. and am Anfang des Satzes, wie: and seiss Conchobar LL. 109 a 42, statt andsin oder isand; auch w. $y d, y$ hat gewöhnlich ein Adverbiale vor sich. Die w. Partikel $y$ mit den pronominalen Suffixen hat mit $y d$ vermutlich nichts zu thun (GC. 421), denn sie ist das ir. do. Neben dem relativischen issid, yssyd giebt es im Altwelschen ein selbständiges Verbum existentiae ('il y a'), das yssit lautet und dem ir. atá entspricht, der Form nach aber mit dem ir. -id (in cid, manid, conid etc.) übereinkommt. So heisst es: yssit teir ffynnawn 'es giebt drei Quellen', Skene 301, 22; yssit imi teir cadeir 'ich habe drei Stihle', 154, 23; yssit a pryderer 'es giebt etwas, was man besorgt', 147,4; yssit ym a lauarwyf 'ich habe, was ich reden möchte', 262,10; yssit rin yssyd uwy (neuwelsch: yssid rin ysydd fwy, MA. 55 a) 'es giebt ein Geheimnis, das grösser ist', 147, 21; darogenwch y Arthur' yssit yssyd gynt 'kündet dem Arthur: es giebt etwas, das zuvor ist', 
Ich nehme also an und zweifle nicht, dass der welsche Stamm byd zur Wurzel $B \hat{I}$ gehört. Seine Konjugation ist fast so vollständig wie die des ir. biuu, biu, Konj. beu, beo, durchgeführt; sein eigentliches Gebiet ist aber das Präsens und Imperfektum: das w. bydwn 'eram' entspricht deutlich dem ir. biinn und kann nicht von der Wurzel $B U$ kommen, die ir. bin, benn : w. bewn : c. ben : arm. benn bildet. Die neugälischen Dialekte fügen dem Imperfekt ein bedeutungsloses $d h$ oder $t h$ ein, das dem welschen $d d$ sehr ähnlich ist, nämlich ir. do bhidhinn, alb. bhithinn, aber manx vein, veign. Der Konjunktiv byddo 'er sei' und der Imperativ bydded 'er sei' scheinen nur der neuern Sprache anzugehören; ebenso die passivischen Formen, wie byddir $=$ ir. bithir 'vivit vir' = vivitur 'man lebt'. Die präsentialen Tempora liefert byd auch den mit bot zusammengesetzten Verben, wie gwybyd 'sci, sciet', gorfyd 'vince, vincet', etc. Dieser Stamm hat kein Präteritum, kein Tempus der Handlung und des Geschehens, doch hat man es im Neuirischen (dobhi = albanogäl. bhà, manx $v a$, vormals $v \hat{e}$ ) analogisch nach dem Präsens bi gebildet. $\mathrm{Zu}$ dieser Wurzel scheint auch der im Mittelirischen aufkommende Infinitiv bith, beith zu gehören, wovon das Substantivum bith : byt : bed 'Welt' nur wenig verschieden ist.

Der andere Stamm, von dem die Konjugation des Verbs both : bot ( $\varphi \dot{v} \sigma \iota \varsigma$, skr. bhûti) ausgeht, im Irischen wieder fast vollständig durchgeführt und hier meist als Kopula gebraucht, zeigt sich am deutlichsten im Perfekt oder Aorist, ir. bói, -bu, w. bu (1. sg. buum), was dem lat. fuit, dem griech. है $\varphi v$ gegenübersteht. Diese Wurzel liefert also das Präteritum, das man von $B \hat{I}$ nicht bildet, so namentlich auch den Zusammensetzungen, z. B. $d y b u$ Skene 197 oder dyfu 'venit', gwybu 'scivit', adnabu 'agnovit', gorfu 'vicit', darfu 'cessavit', hanfu 'exstitit'. Die alte Sprache hat, neben dem Plusquamperfektum buassei 'fuerat', wie das Cornische ein Praeteritum secundarium, das die Bedeutung eines Imperfekts anzunehmen scheint. Es lautet in der 3. sg.

149, 19; auch wohl: yssyd (leg. yssit) wr dylyedawc a lefeir hyn 'es ist da ein vornehmer Mann, der dieses sagt', 124, 17. Dieses it (= ir. -id) hat man auch in: ossit 'wenn es giebt', Skene 188, 4; ot 'wenn es ist' RB. 1, 199; beyt 'wenn es wäre', RB. 1, 175̃; pet ... pet 'sive . . sive', Skene 133, 4 ff. 174, 25; etc. und sehr ursprünglich in: kyt yt uo 'quamquam est ut sit', 286, $20=$ kyt at uo ib. 22, mit merkwürdiger Häufung des Verbum substantivum (gleichsam ir. cid atá ropo). 
buei 'erat' Skene 282, 20. Daneben giebt es auch eine Form auf -at, nämlich buiad 'erat' BBC. 25 b 17, was nach MA. 116 a als bwyat $\mathrm{zu}$ verstehen ist; und dieses bwyat kommt auch sonst in den alten Gedichten vor, Skene 289, 1. 4. 290, 18. Cymmr. 9,65. Die 3. pl. dazu ist buyint 'erant' BBC. $48 \mathrm{~b} \mathrm{2}$, was vermutlich als buyint zu deuten ist. Während buei im Codex Hergestiensis ohne Zweifel zu dem Präteritum bu gehört, muss bwyat vielleicht eher als Imperfektum bezeichnet werden. Beide sind wohl vom Stamme $B U$ abzuleiten, und ebenso das Praeteritum (oder Participium) passivi, ir. both : w. buwyt 'man war', das wiederum in Zusammensetzungen häufig ist. So sagt man: arganvuwyt 'perceptum est' RB. 1, 34, gwybuwyt 'scitum est'; dann aber in kontrahierter Form: gwypwyt; gwanpwyt 'transfossum est' Skene $78,17.122,14$, wnaethpwyt 'factum est' 73, ry vaethpwyt 'nutritum est' 63, 20 (cf. AC. IV. 5, 118). F'benso in der neuern Sprache claddpwyd = claddwyd (Davies p. 98), gwelpwyd, dycpwyd, dywedpwyd (alt und sw. dywespwyt), woneben auch unregelmässig clywsbwyt LA. 117, 14 und clywspwt Cymmr. 9, 77. Offenbar ist also $\boldsymbol{p}=b u, b v$.

Die Wurzel $B U$ bildete wie im Irischen (bid, $b a$ ) so vormals auch im Welschen ein Futurum. Hier lautet der Stamm altwelsch vielleicht boi 'erit' (in hacboi 'excuties' (?), GC. 1056), später aber bi oder pi (aus bvi), und er entspricht, wie es den Anschein hat, dem lat. fio (Brugmann 2,1061). Die Form kommt nur noch bei den alten Dichtern vor, wie: $b i$ 'erit' Skene 62, 9 . 20,11. 123, 11; a ui 'qui erit' 148, 7 f. 222, 6. 231, 12. 236,17; nyt uu nyt vi 'non fuit, non erit' 199, 25; nyth vi 'tibi non erit' 304, 7; häufiger in Zusammensetzungen, wie: dybi 'veniet' RB. 1,119. Skene 111, 8. 128, 10. 292, 7, dyvi 303, 34 (= dybyd 218. 305,1 ), dymbi 'mihi veniet' 211,3 . 238 oder dimbi 205,18 , von dyfod 'kommen'; deupi BBC. 31 a $13=$ deubi 'veniet' Skene 123, 9. 130,1 ; atvi 'iterum erit' 127,9 ff. (== atvyd 296,6 ) von adfod 'wieder sein'; tyrui 'surget' 115, 19 von tyr-fod; dyderbi 'cessabit' 212, 22. 220 (= diderbyd 231, 25. 237,4) von darfod 'aufhören'; dorbi BBC. 31 b 8.14 von dawr 'es betrifft'; gwybi 'sciet' 27 b 9 (=grobyd Skene 161,25) und, last not least : cat a vi ar Byrri auon a Brython dyworpi 'eine Schlacht wird am Flusse Byrri sein und die Britten werden siegen', Skene 237,4, wo $d y$-wor-pi (von gorfod) das $p$ zeigt, das diesem Stamme bi eigentümlich ist oder doch, wie in pwyt, seinen guten Grund hat. Neben dieser 
kurzen Form bi giebt es aber eine vollere mit der Endung des Futurs: biawt 'erit' Skene 228, 3. 229, 13 (cf. MA. 112 b. 113 a) $=$ ir. bid, pl. bit. Ich denke, auch der Imperativ w. bit : corn. bis 'er sei' ist zu diesem Futurstamm zu stellen = ir. bad, vgl. BBC. 10 a 1 f. 13 b 6. 11. Skene 245 (das Gedicht über die bidiau). 261, 19. 305, 16. RB. 1, 147. 246; der Plural dazu lautet bint RB. 1,105. LA. 81,28. Als Zusaminensetzung mit bit ist derffit 'eveniat' Skene 279, 18 zu erwähnen.

Nun handelt es sich darum, von welcher Wurzel die Konjunktive von bot abzuleiten sind. Geht man von der 3. sg. aus, so ist es kaum zweifelhaft, dass w. bo : c. bo, bova 'sit' mit seinem Plurale bont : bons 'sint' dem ir. bo, bat entspricht und dass w. bei : c. bei, feve : arm. be 'esset' das ir. bed, bad ist, beides von der Wurzel $B U$. Darin bestärken uns die Zusammensetzungen mit bot, nämlich gwypo 'sciat' (aus guy-bvo), gwypei 'sciret' (aus gwy-bvei) und auch gwypet 'scito' (aus gwy-bvet) neben gwybydet LA. 110, 10; ebenso dyppo BBC. 45 b 10, deupo 'veniat' Skene 72, 1. 11. 87, 25. Von gorfod lautete der Conj. praes. vormals gorpo 'vincat' BBC. 9 a 14 (aus gor-bvo) und später (nach der Analogie von corff corpus) gorffo, dazu das Impf. gorffei; ebenso darffo, darffei; dyffo Skene 119, 7 neben dyfu, dyfyd 118 etc. Es zeigt sich also, dass der Indikativ (gwoybyd 'sciet', gwybydei 'sciebat') die Wurzel BI 'sein', der Konjunktiv (gwypo 'sciat', gwypei 'sciret') die Wurzel BU 'werden' zu Grunde legt. Die im Neuwelschen aufgekommene Form ('euphoniae gratia', sagt Davies) bae 'esset' (statt bei, bai) scheint sich von pettae 'si esset' aus verbreitet $\mathrm{zu}$ haben; pettawn, pettun 'si essem' geht vermutlich wie pet (nw. ped) auf das Verb ir. tá, atá 'stare' zurück.

Schwierigkeiten aber bietet noch die Fiexion des Conj. praes.: w. bwyf, bych, bo; bom, boch, bont, und corn. beyf byf, by bey, bo; beyn, beugh, bons. Man kann sie mit Hilfe des Irischen kaum heben, da dessen Endungen im Conj. praes. von $B U$ zum Teil verkümmert sind: $b a, b a,-b o ;-b a n, b e d e,-b a t$. Es kommt noch hinzu, dass das Welsche eine alte Flexion des Conj. praes. besitzt, die zu der 1. sg. bwyf stimmt, nämlich: bwym 'simus' Skene 181,6; bwynt 'sint' 112, 6. 124, 21. 212,1. LA. 134, 24. 162,12, bwyn Skene 264, 13; und bwyr 'man sei' Skene 114, 21. Die 3. sg. bwy findet sich in gwypwy 'sciat' Skene 147, $23=$ gwypo. Man kann sich über diese Formen nur mit Vorbehalt äussern. Es ist wahrscheinlich, dass bwy auf *boe beruht und so eine Variante von 
bo ist. Möglich aber auch, dass es gewissermassen dem Präsens wyf 'ich bin', wyt 'du bist', wy 'er ist' 1) etc. analog ist und dass: bwyf ebenso zu bydwn steht, wie wyf zu oedwn 'ich war'; in diesem Falle würde $b w y$ dem irischen $b \dot{e}$ 'sit' von $B \hat{I}$ entsprechen. Jedesfalls dient in der alten Sprache das Präsens wyf der $\mathrm{Zu}$ sammensetzung ebensowohl wie die Formen von bot, z. B. henyw 'exstat', gorwyf 'vinco' Skene 144,2. Es bleibt aber bei alledem zu bedenken, dass sich schon im Irischen Konjunktiv und Futurum mehrfach nahe berühren und fast vereinigen, und so darf man vielleicht auch w. bwy aus dem irischen Futurum bia, w. bwyf aus ir. biam (LH. 1, 110, mit der spätern Var. bum) erklären, wiewohl dieses Futurum von $B U$ erst aus der mittelirischen Sprache nachweisbar ist. Die 2. sg. bych : c. by 'sis' ist gewiss von $B U$ abzuleiten, da sie in der Zusammensetzung pych lautet, wie hanpich BBC. 18 a 2 oder hanpych henpych, jetzt hanffych; aber die altwelsche Form hánbiic (GC. 1063) scheint

1) Diese Form $w y$ (für das gewöhnliche $y w$ oder $w$ wie in derw 'cessat') kommt in einer Stelle des Gododin vor: ny hu $w y$ ny gaffo e neges 'nicht geschickt ist, wer seine Sache nicht erreicht', Skene 78, 15 (MA. 11 a). Vgl. hubwyt 175, 8, hubo 207, 4, hubyd 174, 21. 270, 15 Die Form wy liefert den Schlüssel zum Verständnis des 'Verbs' wyf, wyt, yw. Man darf für dieses Präsens einen pronominalen Ursprung vermuten, da es fast nie an betonter Stelle im Satze steht. Ebenso wie $w y$ zu $y w$, wird auch das verbale Präfix rwy (aus ro-e, ir. ro-d-) zu ryw (RC. 6, 51), und ebenso w. oe 'zu seinem' (= ir. dua, dia) über *vy zu yw (GC. 390); so steht auch moledivo (Skene 272,5) statt moladwy 'löblich'. Demnach vermute ich, dass w. wy, $y w$ : corn. $y u$ : arm. eu 'er ist' dem ir. dáu, do' 'ihm' entspricht; desgleichen wyf : off : of 'ich bin' dem ir. dom, dam 'mir', wyt : os : out 'du bist' dem ir. duit, deit 'dir', und yoch : ough : ouch 'ihr seid' dem ir. duib 'vobis', während $y m: o n: o m p$ 'wir sind' == ir. ammi und ynt : yns : ynt 'sie sind' $=$ ir. at zu dem alten Verbum substantivum gehören. Im Mittelwelschen vereinigt ywch noch die Bedeutungen 'estis' (nw. ych) und 'vobis', aber für die Dative der sonstigen Personalpronomina traten Zusammensetzungen ein, wie $y m$ aus $y$-mi, $y t t$ ans $y$-ti, $y n n$ aus $y$-ni und idaw aus $i$-dd-aw. Die Bedeutung damh 'ich bin', duit 'du bist', do 'er ist' bei präpositionalem oder adverbialem Prädikat ist im Irischen bekannt: a gCill Chreidhe dham 'ich bin in Kilcrea' RC. 7, 68; di feraib Gaidel damsa 'ich bin von den Gälen' RC. 9, 18; can deit? 'woher bist du?' WW. $489 \mathrm{a}$; is ass dam 'ich bin daher' Atk. Gl. 652 a. Allgemeiner ist der (rebrauch dieses dam, duit, do im Welschen geworden, wie in: Lunet wyf $i$ (gleichsam ir. damhsa); hyn gwr wyt 'du bist der ältere' (gleichsam ir. duit); pwy wyt? 'wer bist du?', wofür der Ire sagt: cia thusa? So ist auch w. yttwyf = ir. atá dam. Ob mir. bidam 'ero', var. bidh damh (= w. byddwyf?) RC. 16, 46 und bidat 'eris' (Windisch, Gr. p. 104) eine ähnliche Erklärung zulassen, bleibe dahingestellt. 
wieder auf das Futurum zu deuten, dessen 3. sg. biawt, bi lautet. Darnach ist vielleicht auch diese Annahme berechtigt, dass die Formen bo, bonı, boch, bont aus dem eigentlichen Konjunktive, die Formen bwyf, bych, bwym, buynt aus dem Futurum hervorgegangen sind, und dass sich diese gemischte Flexion erst allmählich festgesetzt hat.

Noch einige andere Formen sind für die Konjunktivbildung im Welschen von Belang. Die Partikel po 'je' würde in diesen Betrachtungen keine Stelle haben, wenn sie wirklich dem lat. quo entspräche, wie in goreu po cyntaf 'je schneller, desto besser'. Aber po mit dem Superlative deckt doch das quo mit dem Komparative nicht völlig. Viel wahrscheinlicher, dass po mit dem Konjunktive $6 o$ 'sit' im Zusammenhange steht ('es sei am schnellsten, so ist es am besten') so wie pei 'wenn wäre' mit bei 'erat, esset'. Was begründet aber die Verhärtung des Anlauts? Man könnte auf die Vermutung kommen, w. po sei das ir. ce bé, ce pé, cia beith 'was auch sein mag'; auch pei liesse sich allesfalls aus cia bed erklären, doch steht daneben die Form ohne $p$, wie vei vei 'gesetzt es wäre, dass wäre', BBC. 9 b 8; bei na bei 'wenn nicht wäre', RB. 1, 189; beyt uei 'wenn sie wäre' 1, 175. Auch ist $z u$ bedenken das $p$ des Imperativs poet 'sit', Skene 78. 91. 96. 109. 110. 125. 129. 178. 179. 203. 259. 299. 304, der neben boet BBC. 49 a 5 ff. (augenscheinlich von dem Konjunktive $b_{0}$ abgeleitet und noch in konjunktivischer Funktion vorkommend, RB. 1, 44.264 f.) und dem schon erwähnten bit besteht. Hier könnte man versucht sein zu vermuten, dass die Verhärtung im Wesen des Imperativs begründet liege (etwa nach Thurneysens Annahme durch eine ausgefallene Interjektion), wie ir. tabair 'gieb' neben dobeir 'er giebt', oder w. tyred 'komm' = dyred, südw. dyre. Aber befriedigender wäre eine Erklärung für po, pei, poet aus der gleichen Ursache. Sie sollte auch auf corn. po, bo, pi und arm. pe 'oder' (GC. 725) anwendbar sein, womit altir. robo (Strachan, Substantive verb p. 34 n.) gleichbedeutend ist.

Dass po, pei, poet aus *bvo, *bvei, *bvoet hervorgegangen sind, dass das $v$ der Wurzel $B U$ das verhärtende Element ist, scheint mir sicher. Aber was hat das latente $v$ hervorgerufen? Die annehmbarste Vermutung scheint mir die zu sein, dass es die Befehl und Wunsch ausdrückende Konjunktivpartikel ist, die im Irischen ro, im Welschen ry, im Cornischen re und im 
Armorischen ra lautet. Weenn sie im weitern Gebrauche, wie auch im Irischen gewöhnlich, die Wirkung der Lenitio hat (wie w. ryvo, GC. 419), so doch thatsächlich nicht in den in Rede stehenden alten, gemeinceltisschen Formen. Ich meine, w. po sei der ir. Konjunktiv praes. rojpo, rop und w. pei das ir. Futurum secundarium ropad. Dass diese Wörter ihr $p$ nur für eine bestimmte Anwendung beibehielten, kann nicht auffallen.

Wo ein $p$ in der Konjugation des Verbum substantivum bot auftritt (der Stamm byd hat es nie), da darf man mit einiger Sicherheit schliessen, dass ess aus der Wurzel $B U$ und nicht $B \hat{I}$ entsprungen ist. Dass das; Präteritum bói: $b u$ dieses $p$ nicht bietet, ist erklärlich, da hie’r von der Substanz der Wurzel nichts verloren geht. Es ist beachitenswert, dass auch die irische Copula (d. i. der Stamm $B U$ ) oft, jjedoch nur im Status contractus, dieselbe Verhärtung des Anlauts zeigt, wie in: ni pam 'non sum', ni $p a$ 'non eris', niptha 'non eras', ni po 'non fuit', ropsa 'fui', ni $p a$ ' 'ne sis', ropat 'sint', während sie in den Formen von der Wurzel $B \hat{I}$, z. B. ni piam 'non vivemus', ni pi 'non vivit' (neben ni bí), ro pia 'vivet' (neben ro bia), wie aus Strachans Sammlungen zu ersehen, seilten ist. Der Umstind, dass das altir. $b$ zum Teil $=p, b b$ und zum Teil $=$ neuir. $b h$ ist, erschwert die Beobachtung. Aber weenn ich nicht irre, so zeigt jenes $p$ der irischen Formen noch Wurzel, indem z. B. ropatt ein ursprüngliches ro-bavat, robvat voraussetzen lässt. Sollte: nicht ein ähnlicher Grund der enklitischen Verhärtung in deer Präposition do liegen (dobeir 'dat', ni tabair 'non dat'), die doch wohl dieselbe ist wie ahd. $z u o$, engl. to? ${ }^{1)}$

Ich halte in diesen Spekulationen inne, um eine Tafel (s. S. 397) vorzulegen, auf der ich die Formen des Verbum substantivum von der zwiefachen Wurzel $B \hat{I}$ und $B U$ je an ihrer Stelle versuchsweise eingesschrieben habe.

1) Der Verfasser des Urkejltischen Sprachschatzes p. 132 scheint es nicht zu billigen. Aber ir. $d$ entspricht doch gewöhnlich hochdeutschem $z$, niederdentschem $t$, z. B. dá : dou zwrei, two; déc: deg zehn, ten; dún:din Zaun, town; dét : dant Zahn, tooth; dér : daigr Zähre, tear; dorn:dworn 'Faust', Zorn; ir. dith 'Ende', Zeit, tidee? (cf. dithugad zeitigen, mit Gegensinn); did Zitze, teat; drial Zagel, towel; dia : duw Ziu, Tyr; damnae : defnydd Zimmer, timber; ir. cride Herz, heart; w. dof zahm, tame; ir. dabach Zober, tab; fodailim ab-zählen, tell; dingim zwingen, twinge; a. a.m. 
DLE FORMEN DES KONJUNKTIVS IM BRITANNISCHEN.

397

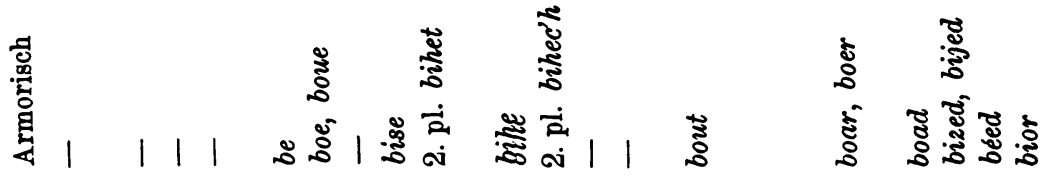

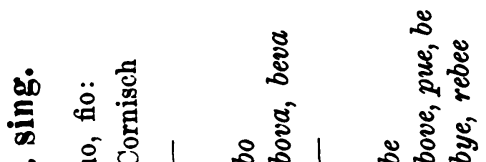

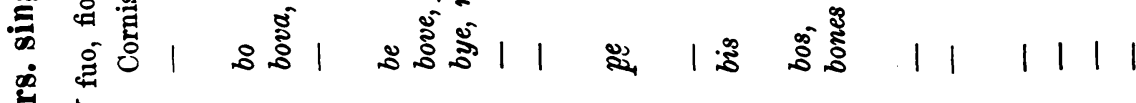

is

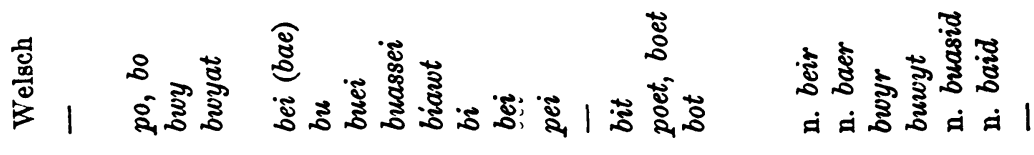

8. की

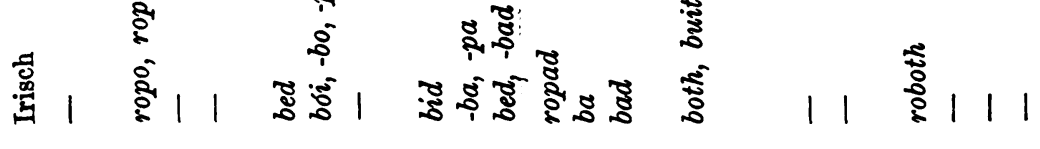

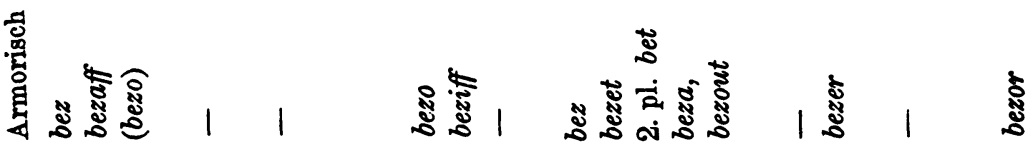

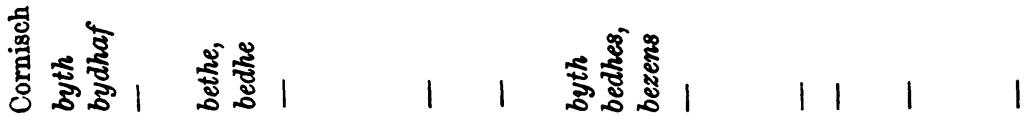

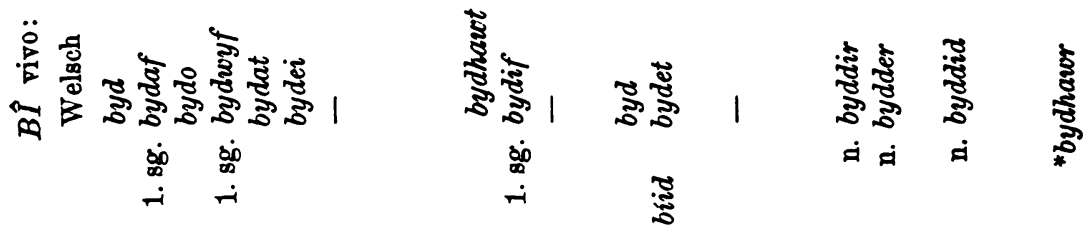

$\stackrel{\circ}{\ddot{\theta}}$

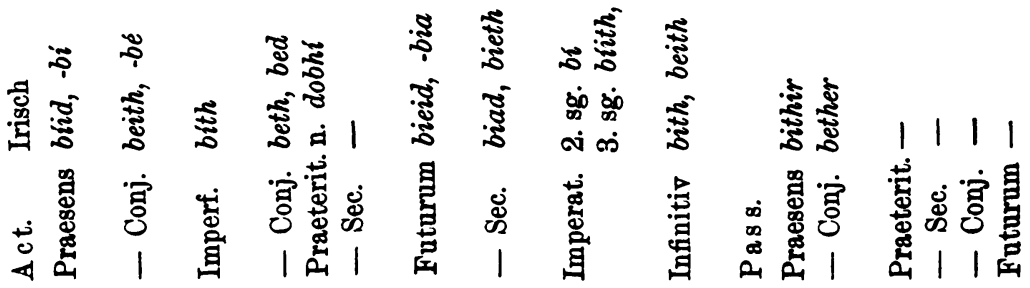


Die allgemeine welsche Konjunktivbildung, wie carho und carhe, scheint sich an die vom Verbum substantivum bot enge anzuschliessen. Sehr bemerkenswert, dass zu carho 'amet' wiederum ein carhwy sich einstellt. Aus dem Konjunktive auf $\bar{a}$, aus dem Wechsel und der Verwechselung des thematischen Vokals, wie etwa w. $o=$ ir. $a$, w. $w y=$ ir. $e a$, lässt es sich schwerlich erklären. Es findet sich: molhwy Skene 161, dalwy 75, 24 (= cynyho 92, 27), llanhwy 117, 10, canhwi BBC. 24 b 3, gorescynhwy 38 b 9, tyfwy Skene 147, 26, gwledychuy 147, 30, guledichuy BBC. 24 b 1. 30 a 16, rybuchvy 38 b 10, dirchafuy 30 a 14, safhwynt Skene 127, 16, rodwy 197, 7 neben rothwy 165, 3, dazu das Impersonale rothwyr 109, 26 (= rothwy yr MA. 81 a), arlaedwy 124, 23, cothuy BBC. 35 b 6 (zu a gaut 41 a 5), nothwy Skene 205, 32, nottvy BBC. 38 b 7, gwnelwy 24 a 15 , rymawy 5 a $1^{1}$ ) und ae harhowe $24 \mathrm{~b} 2$ (= ae harovy $30 \mathrm{a} 17 \mathrm{a}$ ). Neben $w y$ kommt $v e$ vor, wie in eirolve BBC. $16 \mathrm{~b} 12$; und neben $v e$ auch oe, wie in creddoe 27 a 15 ; sodass man endlich auch auf diesem Wege zum einfachen $o$ gelangt. Darauf hat schon Evander Evans die Aufmerksamkeit gelenkt und auch für die 3. pl. die Formen wynt, oynt, oint, oent neben ont nachgewiesen (AC. IV. 4, 147). Diese Übereinstimmung des carho, carhwy, carhei mit gwypo, gwypwy, gwypei scheint kaum denkbar, ohne dass die Konjunktive $b o$, bwy, bei auch auf jene Einfluss gehabt haben, sodass man hier wiederum den Ausdruck des Konjunktivs durch die Wurzel $B U$ erreicht hätte. $\mathrm{Zu}$ dieser Einsicht war Zeuss gelangt, als er sein Futurum *arboim, carboi, carib carab; carbom, carboch, carboint carbont erschloss (GC.1 497), dem er die dann von Ebel als Konjunktive oder Potentiale bezeichneten Formen zuwies. Das $h$ könnte in solcher Zusammensetzung als ein geeigneter Vertreter des $b$ oder $v$ betrachtet werden; jedesfalls hat man im Welschen kehy = key (GC. 139), cahat = caead RB. 1, 208, von cael, arm. cahout, caout=ir. gabháil.

1) Die Form ist m. E. zu erklären aus ry (= ir. ro $)+m$ (suff. 1. sg.) $+\bar{a}$ (d. h. 'ibit') $+w y$ (Endung des Konjunktivs). Deus ren rymawy awen 'Deus domine, adeat me inspiratio' BBC. 8 a 1, korrig. aus rymaw, wofür auch das Impersonale rymawyr 'es werde mir zu Teil' Skene 158, 1. 109,31; Reen nef rymawyr $d y$ wedi, rac ygres rymgwares dy voli. 'Herr des Himmels! zu Teil werde mir dein Gebet (d. h. dass ich zu dir bete, d. h. lass mich zu dir beten), vor Gewaltthat schütze mich dein Lob' (d. h. dass ich dich lobe), 304, 8. Ähnlich wird $a$ auch mit dem Imperf. bei zusammengesetzt: rymafei 'mihi contingebat' Skene 201, 26, wie wnafut 115,15 . 
Eine derartige periphrastische Formation würde der cornische Dialekt erklären helfen, obwohl seine Bildungen von gröberer Textur sind. Konjunktive des Präsens, die den welschen auf $h$ entsprächen, sind wohl nur spärlich nachzuweisen: leuerryf 'loquar', leuerry 'loquaris', peghy 'pecces', carro 'amet' neben caro, tokco 'portet' neben dogo (GC. 583), gehören dahin. Hier bleibt die Komposition mit bot 'sein' nicht auf wenige Fälle beschränkt, sondern ergreift Verba jeder Art. Man hat gothfo 'sciat' (in welscher Schreibung *gwydvo), wothfe = woffe 'sciret' (gleichsam "gwydvei, vgl. gull 'facere' = guthyll, GC. 598), beides von gothros (gleichsam *guydvot, w. gwybod). Man hat aber auch clewfo 'audiat' (w. clywho), wharfo 'accidat' (arm. hoarvezo), perfo 'paret'; und dann auch caruyth 'amabit' (gleichsam *carvyd), taluyth 'rependet', talfens 'valerent' (gleichsam *talvynt). Vgl. GC. 576.

Einen anderen Verlauf hat die Bildung konjunktivischer Formen im Armorischen genommen. Hier findet man in der Konjugation von bot neben den neueren vom Stamme byd abgeleiteten Formen noch solche, die zur Wurzel $B U$ zu gehören scheinen. Sie erscheint indes in der Aussprache $b i$, selbst .im Präteritum biof 'fui' (w. buum), biomp 'fuimus' (w. buam), bioch 'fuistis' (w. buawch), biont 'fuerunt' (w. buant), wozu Ernault im Dictionnaire p. 66 die Variante $e z$ vihont verzeichnet. Nun hat der Conjunctivus praes. neben den von byd abgeleiteten Formen (eig. des Futurs): bezif, ${ }^{1}$ ) bezi, bezo, bizimp, bizint die 2. pl. bihet (vihot, vann. bêê), wozu Ernault im Glossaire p. 229 die neueren Varianten bizhyt, vezot anmerkt. Diese sind der übrigen Flexion von bezif analog gebildet, aber bihet 'sitis' fordert eine Erklärung. Es ist als ein Aorist auf $s$ gedeutet worden (KZ.28, 91), eine Annahme, die das Armorische in einer wichtigen Sache vom Welschen sowohl wie vom Irischen trennt. Ich vermute daher, dass es ein Rest des Futurs von $B U$ ist, zu dem

1) Der Form bezif entspricht im Welschen nicht die neuere, nach Analogie von bwyf gebildete byddwyf, sondern die in einem Verse Taliessins aufbewahrte: ny bydif yn (var. ym) dirwen na molwyf Uryen 'ich werde nicht wieder heiter sein, wenn ich nicht Uryen lobe', Skene 184, 2. 185, 27. 187, 16. $189,10.190,8$. 191, 32 und 196, 15 mit der Variante ny bydaf (cf. 293, 6). Aber auch die Form bydwyf in der Bedeutung 'habeam' kommt schon in diesen alten Texten vor: bydwyf or trindawt trugared 'möge ich von der Dreifaltigkeit Erbarmen haben!' Skene 180, 2, d. i. ir. bidh dhamh (?). 
auch arm. bimp gehört sowie wellsch biawt 'erit', bit 'esto'; so heisst es auch arm. gouzvihet oder gouviet 'sciatis' für w. grvypoch. Das $h$ von bihet ist nur ein anallogisches, wie sich noch weiter zeigen soll.

Eine andere Erklärung sehıe ich auch nicht für das $h$ in dem Conjunct. impf. vihenn, vihes, vihe, vihemp, vihech, das vient wieder ausstösst. Bihe, vann. béhé, ist das Futurum secundarium und entspricht dem w. pei, dem ir. ropad. Die dem w. bydei gleichende Form hat unter den armorischen Dialekten nur das Vannetais boé, wiewohl ihnen der Konjunktiv bezo (vann. bou) geläufig ist. Der trekorische und der venetische Dialekt pflegen nämlich das $z \mathrm{zu}$ elidieren: beet 'sït' statt bezet; anderswo (RC. 17, 287) wird es zu $h$, wie beha 'esse' statt beza. Die neuere Sprache scheint dieses $z$ (w. $d d$ ) im Konjunktive durch $f$ zu ersetzen, wie in ra vefomp = vimp 'simus', ra vefet 'sitis', ra vefont 'sint', ra vefen = ven $(=$ vizen $=$ vijen $)$ 'essem' - ein alter und verbreiteter Lautwechsel.1) Arm. vef- gehört zur Wurzel $B \hat{I}$, aber vi- und vih-zur Wurzel $B U$.

Auch der armorische Dialekt hat manche rohe, den cornischen ähnliche verbale Formen, die auf Komposition mit bot 'sein' beruhen, so wie in gouzvezo 'sciat' (gleichsam *gwydvydo), gouezhimp 'sciamus' (w. gwypwn), gouffenn 'scirem' (gleichsam *guydvewn), goufhemp 'sciremus' (für *gwydveym), gouzvezher (für *gwydvydher) 'sciatur', von gouzuout, gouzout (w. gwybod); cf. GC. 578. Im Infinitive sind solche Zusammensetzungen mit bot sehr gewöhnlich, wie in talvézout, talvout 'gelten', dann auch kerout 'lieben' statt keret, etc. Der Conj. impf. ra garfe 'amaret' ist eine Zusammensetzung mit be 'esset'; die neuere Sprache vermischt ihn noch mit dem Praet. sec. ra garze, ra garje, dessen $z, j$ aus $s$ hervorgegangen ist (RC. 19, 184) = w. carassei, wie arm. bise $=$ w. buassei. Eine Form wie casfe 'odisset' (GC. 521) ist zu neu, als dass sie, wenn es phonetisch möglich wäre, einem irischen Futurum secundarium auf $F$ gleich geachtet werden könnte. Trotzdem hält der von Analogieen überwucherte armorische Dialekt an dem $h$ der Konjunktive (wie in bihet, bihe) zäher fest als selbst der welsche.

Der eigentliche Ursprung dieses Konjunktivcharakters lässt sich aus dem Altwelschen in der Sprache der frühesten Barden-

1) Vgl. gulat Gafis Skeue 214,2 = ir. insi Gaid WT. 302, d. i. Gadis, Cadix. 
gedichte noch erkennen. Es finden sich darin, wie mehrfach bemerkt worden ist, noch deutliiche Spuren der zwiefachen Konjugation, der absoluten und deer konjunkten, im Passiv sowohl wie im Aktiv, jedoch nicht mehr mit der strengen Scheidung des Gebrauchs, die das Irische beobrachtet. Trotz aller Schwankung der Überlieferung kann man fitir die 3. sg. praes. die folgenden Formen aufstellen:

Aktiv: cerit - cari (car), Passiv: ceritor - cerir.

Was das Aktiv anbetrifft, so entspricht die Endung der 3. sg. $t$ (neuw. $d$ ) hier wie überall de:m irischen $d$ (neuir. $d h$ ), wie in carid und nochara, lécid und dollééci. Dass das Schwarze Buch von Carmarthen für dieses $t$ bestïndig $d$ schreibt, ist bekannt. Die welsche absolute Form ist, wie Ev. Evans gezeigt hat (AC. IV. 4, 146), nicht selten: pereid 'es dauert' - ny phara 'es dauert nicht' Skene 289; trengid goluc, ni threing molud, MA. 859 a; kirchid 'greift an' BBC. 46 a 7 ; dyrchenid 'erhebt sich' 41 b 11; gulichid 'wäscht' 46 a 3 ; chwerït 'spielt' Skene 305,3 ; u. s. w. Die vokalisch auslautende konjunkte Form scheint vorzukommen: nym cari 'me non amat' BBC. 2:5 b 1; aber die gewöhnliche, die im Neuwelschen übrig geblieben ist, hat den Vokal abgeworfen und im Stamme oft Umlaut erlitten, wie teifl 'jacit' von taflu, erys 'manet' von aros, etc., aber zufällig car 'amat'. Die ziemlich häufige Endung $a$ der 3. sg. praes., wie doluria (GC.508. AC. IV. 4, 147), scheint sich von den Denominativen auf -hau auf andere Verba übertragen zu liaben. In älteren Fällen der Art kann sich aber sehr wohl der thematische Vokal erhalten haben, den amat hat; es ist selbst wahrscheinlich, dass neben der Form auf $-a$ eine absolute auf -awt gegolten hat: der Futurstamm $b i$ scheint in biawt 'erit' diese Endung angenommen zu haben. Möglicherweise erscheint dieser thematische Vokal als $a$ in gwelattor 'conspicitur' (Skene 303,2) und als $o$ in den alten Formen crihot 'vibrat' (Luxenb.) und brithottor 'variegatur' (BBC. 17 b5).

Auch das Imperfectum hat die absolute Form, wie in bydat Skene 264, 26, gwydiat 'sciebat' neben der gewöhnlichen ohne $t$, wie carei oder auch ceri (AC. IV. 5,117), serui Skene 110, 19, seui 182, 5, gelwi 90,13 , lledei 90,15 , gehabt; beide sind im Irischen $-a d$, -ed, denn hier ist eine besondere absolute Form auf -aid nicht genügend belegt, auch kaum annehmbar.

Das Passiv bildet gleichfalls eine doppelte Form, die eine von der absoluten aktiven cerit aus, wie keritor 'amatur' MA. 177 b 
37; cenitor oder kenhittor BBC. 26 b 7, auch kaintor 'canitur'; kwynitor 'defletur' Skene 280, 24; keissitor 'quaeritur' Skene 157, 12; megittor 'alitur' BBC.31 b 5; ywelitor 'conspicitur' MA. 182 a 3; clywitor 'auditur'; ebenso llemittyor 'calcatur' Skene 305, 4; peritor 'efficitur' MA. 105 b = pervor Skene 237, 7, etc. Daneben kommen aber einige Formen auf etor vor, wie kymysgetor 'miscetur' Skene 181, 72 (cf. ir. commescatar 'miscentur'); cynwyssetor 'continetur' 200, 8; tyghettor 'juratur' (?) 209, 21; dygettaur BBC. 13 a 8, dygetawr Skene 119 'adducitur'; kynbwylletor 'mentio fiet' 200,6. Das letzte Beispiel hat die ausdrückliche Bedeutung des Futurs, wie denn überhaupt schon in den alten Texten die Neigung besteht, dem Präsens die Bedeutung des Futurs zu verleihen, die im Neuwelschen die allein gebräuchliche ist. Die zweite Form des Passivs, die in der neueren Sprache übrig geblieben ist, geht offenbar von der konjunkten aktiven aus: car-ir oder vielmehr cerir, corn. ceryr, cerer, arm. carer, careur.

Nun ist es merkwürdig, dass das Präsens auf -it nicht selten ein $h$ vor der Endung oder die dadurch verursachte Verhärtung des Stammauslauts zeigt, wie Iriuhid ia 'das Eis bricht' BBC. 46 a 7 ; tohid 45 a $13=$ toid ib. 3 'er bedeckt' (cf. Skene 116); aessaur brihuid, torrhid eis 'Lanzen brechen, Rippen werden zerschmettert', 50 a 3; llicrid rid, reuhid llin 'es verdirbt die Furt, es gefriert der See', 45 a 2. 12; gosgupid 'er fegt darüber hin', ib.; mekid 34 a 19 oder meccid 'er nährt' 45 b 3, von magu. Diese Erscheinung ist aus der Form des Präsens unerklärlich, das $h$ muss ihr aus einer anderen zugetragen sein - ohne Zweifel aus dem Futurum, dessen Charakter es ist.

Das eigentliche Futurum der alten Texte lautet in der $3 . \mathrm{sg}$. wieder in absoluter und konjunkter Form:

Akt.: carhawt - carhaw (carho), Pass.: carhator - carhawr. Dass das $h$ hin und wieder fehlt, ist nicht verwunderlich. Indes sind einige dieser Formen vielleicht als Praesentia anzusehen, die als Futura gebraucht sind. Man hat die absolute Form des Aktivs in: parahaud 'manebit' BBC. $50 \mathrm{~b} \mathrm{13}$; briwhawt 'franget' Skene 151, 7, breuhawt 157, 21 ; gwnakawt Skene 150, 24. 30, gwnahaud BBC. 27 b 4.30 b 11 'faciet'; marwhawt 'morietur' Skene 150, 22; gyrhawt 'aget' 124, 22; gwasgarawt 'dissipabit' 229, 14; treiglawt 'percurret' 224, 7; cannawt 'canet' 230, 22; crynnawt 'contre-

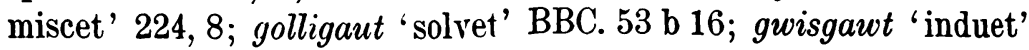
Skene 307; grvledychan't 'regnahit' 221, 23; fflemichawt 'flammabit' 
213, 5; tyfhawt 'crescet' 151, 13; dirchafaud BBC. 27 a 6.30 b 9 , dyrchafaud 24 b 12, dyrchafawt Skene 157, 19, dirchavaud BBC. 31 a 5, dyrchauawt Skene 223 ff. 'ascendet, eriget'; kaffaud 'habebit' BBC. 26 b 6; bithaud BBC. 4 a 1 , bitaud 28 b 7. 29 b 10, bydawt Skene 292, 30, bydhawt 213, 6. 303, 6, bythawt 210, 28. 294, 28 'erit' (cf. GC. 516. 1097. KB. 6, 473. AC. IV. 4, 151). Obwohl diese Form oben dem ir. bieid gegenübergestellt ist, so entspricht sie ihm natürlich formal durchaus nicht (vgl. KZ. 36, 532), denn das w. byd hat die allgemeine Verbalflexion angenommen. Die verkürzte Form ist selten; daw 'veniet' gehört dazu, ferner: a wnaw 'faciet' Skene 150, 9, nys gwnaw 'non faciet' 126, 30; ny chaffau ae hamhevo 'er wird nicht haben, der ihm widerspreche', BBC. 4 b 9. Für -haw erscheint aber schon -ho ganz in der Bedeutung des Futurs, z. B. dideuho, dydeuho 'veniet' Skene 148, 11. 15. Die 3. pl. auf -hawnt hat man in cuinhaunt 'deflebunt' GC. 514; gwnahawnt 'facient' Skene 124, 2; bydawnt 'erunt' 213, 3 .

Von den passivischen Formen ist die vollere die seltenere. Es findet sich: molhator 'laudabitur' Skene 131, 12. 137, 11; canhator 'canetur' 209, 8; gwelhator 'videbitur' etc., auch kwynhyator 'deflebitur' Skene 86, 8; traethatter 'tractabitur' 296, 4. Desto häufiger ist die kurze Form, z. B. dedeuhawr Skene 213, 9, dydeuhawr 212, 22 'venietur'; ryglywhawr Skene 211, 5, ryglywawr 221, 8 'audietur'; galwhawr 212, 26 'vocabitur'; divahaur 'destruetur' BBC. $29 \mathrm{~b} 15$; ffohawr 'fugietur' 126, 34; molhawr 'laudabitur' 165, 18; talhawr 'rependetur' 128, 6. BBC. 16 a 12; carhawr 'amabitur' Skene 117, 19; canhawr 'canetur' BBC. 29 b 9; caffawr 'habebitur' Skene 235, 27. Dass das $h$ in diesen Formen nicht willkürlich ist, zeigen solche wie: etmyccawr BBC. 29 b 13, dydyccawr Skene 166, 9 f., mettawr, dottawr 136, 28, crettawr u. a. m., die Dottin, Les désinences verbales p. 169, verzeichnet. An der ursprünglich futuralen Bedeutung ist kein Zweifel: kirn a ganhawr, briuhaud llurugev 'Hörner werden geblasen werden, Panzer werden brechen', BBC. 29 b 9.

Die Flexion des Conjunctivus praesentis mit ihrem durchgehenden $\bar{o}$ ist, wie schon Ev. Evans gesehen hat, mit dem Futurum auf -haw zusammengefallen. Das $h$ hat der Konjunktiv carho, carhwy daher nach aller Wahrscheinlichkeit einzig und allein von dem Futurum auf -haw. Elenso weist der Conj. praes. pass. carher (corn. carer, arm. carer, carher, carheur, dann auch caror, carfer) auf das Futurum auf hawr und dieses wird schon in alter 
Zeit als Konjunktiv gebraucht, z. B. eiryaul a garawr hawdweith 'das Bitten eines, den man liebt, ist leicht', Skene 308, 3, für sonstiges a garher und heutiges a garer. In einem südwelschen Dialekte ist, wie Dottin p. $174 \mathrm{f}$. anführt, die Endung -awr ganz an der Stelle des sonstigen -er noch üblich. Erscheint eine solche Form ohne $h$, wie hier in carawr, so würde man sie von einem alten Konjunktiv des Aktivs cara ableiten können; durch das $h$ wird sie als Futurum gekennzeichnet. Die Flexion des Conj. praes. von $B U$ hat vielleicht die Norm gegeben, namentlich für die 3. sg. in allen Dialekten: w. carho, corn. caro, arm. caro, vann. cârou, vielleicht auch für die 3. pl. (ont:ons:ont); aber in der 1. und 2. pl. zeigt sich im Welschen (om, och $=$ corn. yn, ough) vielleicht der Einfluss des Futurs. Das Armorische hatte vormals im pl. auch Formen auf o, z. B. labourhomp 'laboremus', casont 'oderint'; aber sie sind durch solche auf imp, it (et), int verdrängt worden.

Mit dem $h$ des Futurs hat man, wie es nach dem oben bemerkten scheinen muss, ein Präsens-Futur auf -hit gebildet, das im Welschen, als Futur oder Konjunktiv praes., selten ist, z. B. bythit (d. h. byd-hit) 'erit, sit' Skene 213, 7; dottint 'dabunt, dent' BBC. 29 b 8 . Als 1. sg. gehört dazu bydif, das vorhin erwähnt ist, und: ew kuynhiw iny wuiw in hervit hon 'ich werde ihn beklagen, so lange ich in diesem Verhältnis bin', BBC. 29 b 8; doch scheint diese Endung if: corn. $y f$ : arm. iff auch zu -hawt die 1. sg. zu bilden. Im Armorischen ist dieses Präsens-Futur für Futur und Konjunktiv praes. die gewöhnliche Form, z. B. cleuimp 'audiamus', veohimp" vivamus', neben clevhet ' audiatis', querhet, carehét' ametis' und, nach Analogie dieser Formen, bihet 'sitis'.

Ist also der Conj. praes. carho gewissermassen von dem Futurum abzuleiten, so müsste für carhei sich ein Futurum secundarium darbieten. Eine ältere Form dafür als eben dieses carhei, arm. carhe, vann. câréhé ist nicht bekannt. Das arm. bihe 'esset' hat sein $h$ wiederun nach der Analogie von carhe. Ebenso muss auch das Passiv des Conj.impf. cerhit auf einem Futurum sec. beruhen; das neuarm. carfet, carfed ist dagegen eine Zusammensetzung mit béed, der entsprechenden Form des Verbs $B U$. Denn dieser Dialekt bew'ahrte den Konjunktivcharakter im Plural der gesamten Konjugation mit bemerkenswerter Treue, als das Welsche und Cornische ihn längst aufgegeben hatten, wie 
am Verb gra- (von for-ag-, w. gwneuthur) erläutert sei: groahimp 'faciamus', grehet 'faciatis', grahint 'faciant' (w. gwnahont BBC. 31 a 15), neben dem Imperative greomp, gret, graeñt; grahemp 'faceremus', grahec'h 'faceretis', grahent 'facerent' (w. gwnaem, -aech, -aent); graer (w. gwneir) 'fit', graher (w. gwnaer) 'fiat'; graet 'factum est', grahet 'fieret'. Darf man die Formen von arm. gra, w. guna in unmittelbaren Zusammenhang bringen mit w. $a$ 'ibit' $=$ ir. roa, wozu der Plural ri-sam, ri-sta, ri-sat lautet, so ist der von Vendryes aufgestellte Satz für diesen Fall erwiesen. Aber es will fast scheinen, als sei grahint, grahent etc. nicht anders zu beurteilen als rohint 'dent', rohent 'darent', d. h. es sind Analogieen nach carhint, carhent, wo die Ableitung vom Futurum auf $s$ nicht wohl möglich ist.

Aber dennoch giebt uns das Irische die letzte und wichtigste Aufklärung über diese Bildungen. Die welschen Futura oder Konjunktive auf $H$ entsprechen offenbar den irischen Futuren auf $F$ oder $B$, nämlich:

w. carhawt, cerhit - carhaw, carho 'amabit, amet' dem ir. carfid, no charfa;

w. carhei 'amaret' denı ir. carfad;

w. carhator - carhawr, carher 'amabitur, ametur' dem ir. carfaidir, no charfider;

w. cerhit 'amaretur' dem ir. carfide.

Wahrscheinlich findet auch w. bydif, arm. bezif vivebo 'ero, sim' in der irischen konjunkten Form no charub, dolléciub seine Erklärung. Die durch das $h$ des Futurs im Welschen veranlasste Verhärtung einer auslautenden Media der Wurzel (wie mekit 'alet' von magu 'alere') hat eine ganz merkwürdige Parallele im Vulgäririschen: hier spricht man fágaidh 'er verlässt' wie fāğe, aber fāgfaidh 'er wird verlassen' wie făǩ̆; lúbaidh 'er

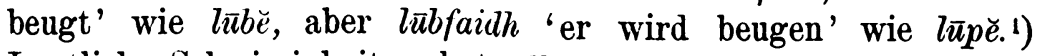
Lautliche Schwierigkeiten hat alles dies überhaupt nicht, denn auch im Irischen schwankt die Vokalisierung der Formen, die wiederum nur Umschreibungen mit dem Futurum von $B U$ darstellen, nämlich bid, -ba und seinem Secundarium bed, -bad.

Und hier erweitert sich der Blick über die Grenzen des celtischen Gebiets ins Italische. Denn man kann es nicht dem

') Vgl. F. N. Finck, Die araner mundart 1, 140. 142. 
Zufall beimessen, wenn sich die mit der Wurzel $B U$ zusammengesetzten Formen in beiden Sprachen so überraschend entsprechen, und ir. carfid = w. carhawt = lat. amabit, ir. carfad = w. carhei $=$ lat. amabat, ir. carfaidir $=$ w. carhator = lat. amabitur sind gewiss des gleichen Ursprungs. Der Einklang der beiden Sprachstämme scheint vollkommen zu sein, und ich muss noch eines hinzufügen.

Obwohl ich den gelehrten und belehrenden Untersuchungen über den Ursprung des celtischen Passivs zu folgen suchte, so ist es mir doch nicht gelungen, mir daraus eine Überzeugung anzueignen. ${ }^{1)}$ Es scheint festzustehen, dass diese Bildungen auf $R$ nicht sehr alt, nicht indogermanisch sind; dass die Deponentia jünger als die Passiva sind; dass diese Formen sich zum grössten Teile analogisch über die ganze Konjugation ausgebreitet haben und dass man, nach ihrem Ursprunge forschend, immer wieder von der 3. Person ausgehen muss. Und in der That, das ist ja überall aktive Flexion mit dem Zusatze eines $R$, das sich mit wechselndem Vokale anhängt. So im Altirischen: dober 'dat', dober-r oder dober-ar 'datur'; doberat 'dant', dobert-ar 'dantur'; oder: gaibid. 'capit', gaibthi-r 'capitur'; gabait 'capiunt', gaibti-r 'capiuntur'. Dieses $R$, wie man wohl erkannt hat, das eigentlich Wichtige in der ganzen Erscheinung, ist verschieden gedeutet worden, bald als aus $S$ entsprungen, bald als eine Endung der 3. pl. und bald als aus einem vokalisch auslautenden pronominalen Suffix gekürzt. Verzeihung, wenn ich, solchen Weisungen widerstrebend, einen fast unbetretenen Weg einschlage und eine ganz andere Lösung des Rätsels für möglich halte. Meines Erachtens liegt das italoceltische Passiv weniger in der Form als in der Syntax. Zeigen die erwähnten Formen die 3. Person sg. und pl., so könnte die Endung $R$ das Objekt sein, wie in gaibth-i von gaibid oder gabt-ait von gabait (KZ. 28, 319); nichts aber hindert, dass es das Subjekt sei, das sich der 3. sg. und pl. anhängt wie etwa die Suffixe $s$ und $e$ der relativen Formen: $b i-s$ 'qui est', bit-e 'qui sunt'. Keine andere Auffassung ist möglich im lat. it-ur 'man geht' und im altlat. potest-ur 'man kann', oder possit-ur, poterat-ır, posset-ur. Ich vermute dem-

1) Vgl. F. Stolz, Lateinische Grammatik ${ }^{3}$ (München 1900), p. 157 ff.; R. Thurneysen in KZ. XXXVII (1900), p. 92 ff. (während des Druckes dieses Aufsatzes erschienen). 
nach in dem $R$ ein nominales oder auch pronominales Subjekt zu der aktiven Verbalform. Da liegt es nahe zu prüfen, ob diese beständig konsonantisch auslautende Endung nicht etwa mit dem lat. Worte vir, ir. fer, w. gwr, 'wr im Zusammenhang steht. Dann heisst ir. bithir, w. byddir, lat. vivitur (aus bith-fer, bydit-wr, vivit-vir) 'der Mann lebt, man lebt'. Diese Vermutung hat schon vor vielen Jahren J. Rhŷs ausgesprochen (KB. 7, 57), wenn auch, so viel mir bekannt, ohne sie zu begründen oder zu verfolgen. Sie ist gewiss nicht ganz unwahrscheinlich, wo wir das deutsche man (aus der mann) und das französische on (aus homo) vor uns haben. Auch für die Möglichkeit der Verkürzung eines solchen Ausdrucks sind on und man lehrreich, für welches letztere das Mittelhochdeutsche und Niederländische geschwächte Formen wie men, me darbieten (J. Grimm, Grammatik 3,8). Ferner ist man schon in der alten Sprache ein Collectivum, das sich sowohl mit dem Singulare seines Verbs als mit dem Plurale verbindet (Grimm 4, 221). Dasselbe dürfte man im urceltischen Gebrauch für das ir. fer in Anspruch nehmen.1) Wen die formale Erklärung des $R$ aus Personalendungen befriedigt, der wird ja freilich einer solchen Hypothese keine Beachtung schenken. Den Beweis ihrer Richtigkeit vermag auch ich nicht zu erbringen; daher soll fer 'vir' hier nur als eine Formel gebraucht werden, als ein bekanntes Concretum für ein unbekanntes Abstractum. Darf ich es nach dieser Verwahrung ausführen, so suche ich die Genesis der Formen auf $R$ folgendermassen zu verstehen.

1) Es sei daran erinnert, 'dnss fer : gwr 'Mann' in den celtischen Dialekten eine allgemeine Bedeutung hat, die einem Pronomen demonstrativum gleichkommt, wie ir. a fir do chumm in cruinde 'o Mann, der die Welt geschaffen' LB. 186, 13; albanogäl. am fear a thuair $i$ 's leis còir oirre, 's gu bheil ise ann an solas nan gràs 'der Mann, der sie genommen, hat ein Recht anf sie, so ist sie denn im Troste der Gnaden', Glenbard coll. p. 177 in einer Totenklage; w. mynn y gwr an gunaeth 'bei dem Manne, der uns geschaffen', RB. 1, 190. Die gälische Volkssprache geht in diesem Gebrauche viel weiter, wie man aus einigen Beispielen ersehen mag: 's an tsaoghal 80 no 's an aththear 'in dieser Welt und in dem andern Es', d. h. in der andern, Campbell, Tales 2, 284; am fear eile 'der andere Mann', d. h. hier der Wolf, 3, 105; sonst gesagt von einem Pferde, Nicholson, proverbs p. 342; von einem Vogel; 359; von einem Kuchen, 162; fear ùr 'ein neues Es' d. h. ein neues Haus, Highland Monthly 2, 46; fear mo laimhe deise 'das Es', d. i. das Schwert 'fur meine rechte Hand', Campbell 3, 343; fear de na bioran 'ein Es', d. h. einer, 'von den Spiessen', Mac Innes, Tales p. 80. 
Die celtischen Passiva würden von der Vorstellung ausgehen, dass zu der passivisch ausgedrückten Handlung zwei gehören, das Subjekt (d. i. fer 'vir'?) und das Objekt, das im Akkusativ steht. Das Irische erweist sich darin ursprünglicher als das Lateinische, das mit dem Passiv den Nominativ verbindet; jedoch hat Ennius noch die ältere Konstruktion: vitam vivitur 'man lebt ein Leben'. Es liegen nämlich dem celtischen Satze drei Formeln zu Grunde: caedit vir d. h. caeditur 'man tötet', 'er wird getötet'; caedit vir virum oder me, te, nos, vos; und caedunt viri viros 'Mann kämpft gegen Mann; Männer töten Männer'; 'man tötet den Mann, man tötet die Männer'. So ist das paradigmatische ir. carthir 'amatur' (w. ceritor) $=$ carid 'amat' (w. cerit) + fer 'vir' (w. wr); ir. caritir 'amantur' = carit 'amant' + fer 'viri'; carfaidir 'amabitur' (w. carhator) = carfid 'amabit' (w. carhawt) + fer 'vir'; carfitir 'amabuntur' = carfit 'amabunt' + fer 'viri'. Das Futurum secundarium ist participial und hat daher kein $R$. Das Britannische kennt nur die ursprünglichere Formel amat vir, amabit vir, kein amant viri. Fehlt einem solchen Ausdrucke das Objekt, so ist er intransitiv oder neutral. Die Zweideutigkeit, die er unter Umständen hätte haben können, wird von der Sprache auch sonst zugelassen; ist es doch z. B. nicht ohne weiteres zu sagen, was in einem Satze wie neuir. an bhean do bhuail an fear oder an bhean nár bhuail an fear oder 'die Frau, die das Kind schlug' etc. das Subjekt und was das Objekt ist. Der Vokal vor dem $R$ schwankt im Irischen zwischen $i, e$ und $a$, je nach dem Charakter der durch Vokalharmonie bestimmten, unter den einheitlichen Wortaccent gestellten Form; das Welsche bewahrt in den vollen Formen (ceritor, carhator) das dunklere o (= lat. $u$ ), in der geschwächten (carhawr) $w$, und beides würde aus gwr, wr verständlich sein. $\left.{ }^{1}\right)$

Wie mit dem erwähnten Prïsens und dem Futurum auf $B$ verhält es sich auch mit den andern irischen Tempus- und Modusformen: sie sind die 3. sg. und pl. Activi mit dem Anhange $R$ (fer), wie z. B. berid 'fert', berir (statt berth-ir) 'fertur'; bérid 'feret', bérth-ir 'feretur'; adchi (statt *adchid) 'conspicit', ad-

1) Dürfte man dem ir. Suffixe ar, er, bar der Abstracta, wie oinar 'Einheit', coicer 'Fünfheit', nónbar 'Neunheit', in urceltischer Sprache die Bedeutung und die Selbständigkeit des pronominalen 'man' zuerkennen, so würde es vielleicht eine noch leichtere Erklärung des vermuteten subjektivischen $\boldsymbol{R}$ gewähren als fer, mit dem es freilich nicht identisch ist. 
chith-er 'conspicitur'; carad 'amato', carth-ar 'amator'; dlésit 'merebunt', dlessit-ir 'merebuntur', u. s. w.

In den Formen gleicht das irische Deponens durchaus dem Passiv: es sind in allen Personen die aktiven Endungen mit angefügtem $R$; z. B. carid 'amat', labrid-ir 'loquitur'; nochara 'amat', -labrath-ar 'loquitur'; -molat oder -molat-ar 'laudant'; carais (statt *caraist) 'amavit', labrist-ir 'locutus est'; carsit 'amaverunt', labrisit-ir 'locuti sunt'; carfid 'amabit', sudigfid-ir, noshudigfedar 'ponet'; seiss oder siass-air 'constitit'; tésit 'ibunt', fessit-ir 'scient'; aithgén 'agnovi' = w. adwaen, rothet-ar 'scio' = w. gun (statt *gwend); aithgéuin 'agnovit' = w. edwyn, rofhit-ir 'scit' = w. gwyr, mit Verlust des $t$ wie in cerir neben ceritor. Nur in der irischen Formation der 3. Person praes. weicht das Deponens vom Passiv ab: ersteres knüpft die Endung vokalisch an, wie in cairigedar 'reprehendit', letzteres stösst den Vokal aus, wie in cairigthir 'reprehenditur'; ebenso ni labrathar 'non loquitur', aber labairthir 'dicitur' (Sächs. Ges. d. Wissensch. 23, 483). Vielleicht darf man hierin eine Andeutung des verschiedenen Sinnes erkennen, den das $R$ des Deponens notwendigerweise hat. Wenn man das $R$ des Passivs als das verbale Subjekt vir : fer 'man' auffasst, so steht doch dieses selbe vir : fer 'der Mann, die Person, der Einzelne' in den deponentialen Formen als Apposition, gewissermassen in der Bedeutung von 'viritim': sechur sequor 'ich folge einzeln, in Person'; labrur loquor 'ich rede meinerseits', midiur meditor 'ich denke bei mir', mitter (statt midither) meditaris, vormals meditarus ${ }^{1}$ ) (statt ${ }^{*}$ meditas-ur), etc. Die 2. sg. praes. depon. labrither 'loqueris' lässt auf eine aktive Endung -ith schliessen, die vermutlich die in den britannischen Dialekten noch vorkommende ist, nämlich altw. lleferyd : corn. leuereth : arm. leuerez 'du sprichst' (AC. IV. 4, 143). I)ass das deutsche man auch die 1. Person, den Redenden selbst, und die 2. Person, den Angeredeten, bedeuten kann, brauche ich Kennern der Sprache nicht zu sagen. Ohne Zweifel liegt ein feiner Unterschied darin, wenn das Irische die angeredete Mehrheit nicht als ein 'man' bezeichnet, sondern in der 2. pl. (sechid) die aktive Form schlechthin gebraucht, während das Lateinische (sequimini) sie participial ausdrückt.

1) Vgl. W. M. Lindsay, The Latin Lauguage (Oxford 1894), p. 534. 
Durch die Apposition vir : fer (ich verfolge die analogische Bildung in den andern Formen nicht weiter) wird das aktive Verb zunächst ein intransitives: es drückt den Zustand oder die Thätigkeit aus, die in dem Subjekte bleibt, vor allem die geistige Thätigkeit. Amat patrem ' $\mathrm{er}$ liebt den Vater', aber ohne Objekt amat, est in amore 'er ist im Zustande des Liebens'; amatur 'er in Person, seinerseits, in sich, ist im Zustande des Liebens' (ir. *caridir) könnte im Lateinischen, wie die Deponentia beweisen (hortatur filium), noch aktiv gewesen sein; beim Mangel eines Objekts liegt die passive Bedeutung nahe (ir. carthir) und in amatur a patre ist eine andere ausgeschlossen. Gewiss hat das Lateinische vormals ein Medium besessen, wie man aus sequere $=\varepsilon \pi \varepsilon(\sigma) o$ folgert. Aber wenn lateinischen und celtischen Deponentien in andern indogermanischen Sprachen Media derselben Verba gegenüberstehen (wie lat. sequitur, ir. sechidir $=$ sanskr. sacate, griech. हैं $\varepsilon \tau \alpha \iota)$, so beweist das doch nur für die Bedeutung, aber nichts für die Form. Mir ist es wahrscheinlich, dass das Passiv und das Deponens im Celtischen und im Lateinischen vom Aktiv abgeleitet sind. Daraus würde aber folgen, dass die Endung $R$ subjektivische oder appositionale Bedeutung hat. Ihre Erklärung durch das ir. fer 'vir, viri' ist freilich nur ein Behelf um den geforderten Sinn zu voranschaulichen. Est quadam prodire tenus, si non datur ultra.

Berlin.

Ludw. Chr. Stern. 